\title{
Réflexions à propos de la Conférence du Professeur Robert C. Merton
}

\author{
par Jean Dromer*
}

La finance est un domaine où la recherche universitaire a beaucoup apporté durant ces dernières années. La réflexion académique répond aux préoccupations des practiciens et parfois même les précède. D'ailleurs, la référence à nos amis chinois est à la fois une autorité et une preuve.

Les travaux du Professeur Merton font autorité. L'exposé qu'il vient de nous faire révèle la richesse et la précision de sa pensée non seulement théorique mais aussi ouverte sur le monde réel. Nous devons l'en remercier. Il est certain que la finance se prète bien à la recherche théorique car c'est l'endroit où le temps et l'incertitude s'entremêlent inextricablement. La préoccupation de tout investisseur, et notamment d'une compagnie d'assurance, est triple: c'est la sécurité, la rentabilité et la liquidité des placements. L'optimisation de ces trois facteurs peut être recherchée théoriquement pour éclairer et orienter l'attitude des practiciens.

Les modèles financiers en temps continu qui sont, si j'ose dire, la spécialité du Professeur Merton, permettent de mieux éclairer les choix des investisseurs dans la durée. Certains problèmes très pratiques, comme la recherche de couverture ou l'évaluation des options peuvent être résolus par l'application de techniques fondées sur l'analyse en temps continu.

Le gestionnaire ne peut ignorer les nouveaux instruments financiers car, quand bien même ne s'intéressait-il pas à ces nouveaux marchés (options, «futures», convertibles, warrants, ...), leur seule existence a un effet sur les marchés où se négocient les produits (actions, indices boursiers, obligations, devises, matières premières, ...). Qui plus est, il se voit offrir des instruments susceptibles de modifier mais surtout d'améliorer la gestion des portefeuilles. Cependant, la complexité de ces nouveaux produits exige de connaître les fondements des théories qui permettent leur évaluation afin de pouvoir maîtriser et non pas subir les risques inhérents à toute activité de gestion utilisant ces instruments.

Je me permets de descendre de la théorie, si séduisante et instructive soit-elle, vers un domaine que je maîtrise moins mal, celui de mes préoccupations professionnelles en matière de placements.

\footnotetext{
*Président, V.A.P., Paris
} 
L'assurance est un métier financier, dont la matière première est l'argent, et dont la variable est le temps. Sa spécificité (par rapport à la banque par exemple) est qu'elle s'intéresse surtout au temps. C'est sur une longue distance qu'elle doit limiter ses risques et protéger sa substance, tout en recherchant la meilleure performance possible.

Le bilan d'une compagnie d'assurance-vie fait apparaître au passif les engagements visà-vis des assurés, appelés provisions mathématiques, et à l'actif les valeurs mobilières et immobilières qui en constituent la couverture. Ces placements doivent permettre de mener une gestion sur une période longue, garantissant un revenu suffisant pour répondre aux engagements pris, en particulier en terme de taux minimum garanti, quelles que soient les évolutions des marchés financiers.

Une règle de bon sens qui en découle est la répartition des risques. La meilleure protection sur le long terme est la structure du portefeuille. L'UAP réalise cet équilibre entre les divers instruments en ayant dans ses placements à peu près $60 \%$ d'obligations, $20 \%$ d'actions, $20 \%$ d'immobilier. Donc plus de la moitié de revenus dit fixes et moitié de revenus dit variables. Ces derniers permettent, par la réalisation plus ou moins forte de plusvalues, de lisser la rentabilité de l'ensemble.

Encore faut-il que le choix des valeurs ait été judicieux : c'est-à-dire fait avec discernement, en temps opportun.

La gestion du temps est donc nécessaire. L'objet essentiel d'un marché à terme d'instrument financier, comme le MATIF, est de permettre la gestion du risque de taux d'intérêt. Le risque existe pour les actifs et pour les passifs, aussi bien à la baisse qu'à la hausse des taux.

L'un des risques majeurs pour une compagnie d'assurance est de détenir des actifs trop courts par rapport à ses engagements. Ce danger se situe notamment du côté d'une baisse des taux. Le fait de ne pas pouvoir investir à l'instant opportun a des incidences regrettables. Si un portefeuille est constitué d'obligations plus courtes que les engagements et si les taux d'intérêt baissent, les coupons desdites obligations seront réinvestis à des taux moins élevés. D'autre part, si les taux sont restés bas au moment de la récupération du capital, celui-ci sera lui-même réinvesti à un taux plus faible. Le recours aux techniques en temps continu pour gérer ce risque apparaît utile. La gestion de valeurs mobilières recherche une performance relative à un objectif. C'est grâce aux nouveaux marchés que les gestionnaires pourront mieux faire coïncider leur comportement en regard des objectifs qui leur sont assignés. Mais ces marchés ne peuvent pas être regardés comme des marchés de spécialistes; les gestionnaires doivent en acquérir la connaissance et la pratique sous peine de perdre rapidement une part de leur compétitivité.

La fonction d'une gestion en continu pour un investisseur institutionnel c'est la couverture des risques plus que l'arbitrage et surtout que la spéculation. Il est permis de se demander si une réflexion attentive et lucide des données de base du marché n'est pas d'une meilleure gestion que la couverture systématique d'un risque mal apprécié.

Cette constatation me conduit à formuler quelques interrogations hâtives :

1) Le marché secondaire devient une fin en soi et non plus seulement l'auxiliaire du marché primaire. Sa liquidité y gagne beaucoup, ce qui est un point très positif. Mais n'est-ce pas au prix d'une multiplication des transactions dont le bien-fondé n'est pas toujours évident? 
2) Les horizons de gestion ne cessent de se rapprocher. Les nouveaux instruments financiers sont le plus souvent des instruments à court terme. Or, c'est le placement à long terme qui a seul une véritable signification et une véritable utilité économiques. La performance sur des périodes de plus en plus brèves devient le souci majeur et le critère le plus usité des gestionnaires, et la revendication des porteurs de titres. Certes, l'important est que les émetteurs de papier puissent collecter des capitaux à long terme, mais est-il bien de l'intérêt des porteurs de s'en défaire aussi vite?

3) Dans des laps de temps toujours plus courts, la spéculation domine l'investissement. Ce qui ne serait éventuellement qu'un accident de parcours dans un placement long devient une erreur de gestion qu'il faut corriger sans délai. Est-ce la meilleure méthode pour gérer une épargne investie?

4) L'érosion constante des marges (notamment en France depuis la libre négociation des courtages) pousse les intermédiaires à multiplier les opérations. Est-ce une raison suffisante pour légitimer, au moindre écart de cours, des négociations qui, dans une optique de placement à long terme, ne s'imposeraient nullement ?

5) La griserie de l'innovation technique des nouveaux produits financiers requiert une agilité et une jeunesse d'esprit qui ne sont peut-être pas toujours compatibles avec la réflexion macro-économique et la maturité de jugement?

Ces cinq interrogations, sommairement exprimées, se ramènent à une question fondamentale: La gestion des portefeuilles est-elle vraiment rendue plus efficace par l'usage intensif de nouveaux instruments financiers? La réponse est certainement positive dans la théorie, mais dans la pratique ne faut-il pas se montrer plus circonspect?

La création d'un nouveau marché ne fait pas disparaître les moyens utilisés antérieurement pour en combler l'absence. Le contrat notionnel, par exemple, n'a mis fin ni aux émissions à taux variable, ni aux opérations à terme de gré à gré, ni à l'usage des rémérés. Cela se comprend dans la mesure où un contrat standard ne répond pas à tous les besoins potentiels. Mais une autre explication n'est-elle pas que les spécialistes anciens ne se résignent pas à abandonner leur sphère d'influence?

L'usage d'un nouvel instrument, au moins pour un temps, peut entraîner des conséquences opposées à l'objectif poursuivi. Ainsi, la possibilité de couverture contre le risque de taux réduit l'efficacité de la politique monétaire conduite par les pouvoirs publics. De même (et c'est fondamental), l'usage systématique de la couverture interdit les plus-values qu'une gestion plus judicieuse encore que plus risquée, aurait permis d'obtenir. On a remarqué aussi que les écarts de cours sur le MATIF accentuent les tensions qu'il est censé atténuer. La spéculation, dans un premier temps, exagère les "pointes» même s'il est vrai qu'elle les atténue finalement puisque toute position doit être dénouée par une opération inverse.

Les nouveaux instruments financiers ne procurent-ils pas, parfois, que des illusions de sécurité? L'on confond souvent la couverture avec une assurance. Il n'y a pas d'assurance possible pour des risques qui, s'ils viennent se concrétiser, toucheront en même temps tous les porteurs d'un certain type de biens. La mutualisation de ces risques, base de la fonction d'assureur, ne sert ici à rien. En cas de hausse des taux par exemple, tous les détenteurs d'obligations à taux fixes seront frappés le même jour. Qui pourrait les garantir dans une telle circonstance? 
Il se peut d'ailleurs que la spéculation se dérobe. Lorsque le risque devient une certitude, on ne trouve plus personne pour l'assumer, ou bien alors à des conditions prohibitives.

Les quelques considérations qui précèdent n'ont pas pour objet de jeter le discrédit sur les nouveaux instruments financiers. Je suis très conscient des remarquables progrès techniques que constitue leur création, et dont nous sommes redevables pour la plus grande part à des chercheurs tels que vous, Professeur Merton. Il me paraît seulement utile de susciter, de la part de leurs utilisateurs, une réflexion plus approfondie sur la meilleure façon d'en faire usage. Comme la langue d'Esope, ils peuvent être la meilleure ou la pire des choses.

Une technique ne constitue pas, en soi, une politique; elle n'en est que l'instrument. La gestion d'une trésorerie, ou a fortiori d'un patrimoine, requiert d'autres qualités que la dextérité dans l'usage de techniques sophistiquées. Ne mélangeons pas l'objectif et les moyens. Ne nous laissons pas fasciner par le succès de telle ou telle individualité. Rappelons-nous que les Compagnies d'assurances sont d'abord fondées sur le collectif et associent finances et probabilités. C'est la raison pour laquelle aussi je dirai au Professeur Merton merci pour le «temps continu»... mais réfléchissons encore ensemble «au temps adapté». 